\title{
Temporal Localization of Fine-Grained Actions in Videos by Domain Transfer from Web Images
}

\author{
Chen Sun* Sanketh Shetty ${ }^{\dagger}$ \\ *University of Southern California \\ \{chensun,nevatia\}@usc.edu
}

\author{
Rahul Sukthankar ${ }^{\dagger}$ Ram Nevatia* \\ †Google, Inc. \\ \{sanketh,sukthankar\}@google.com
}

\begin{abstract}
We address the problem of fine-grained action localization from temporally untrimmed web videos. We assume that only weak video-level annotations are available for training. The goal is to use these weak labels to identify temporal segments corresponding to the actions, and learn models that generalize to unconstrained web videos. We find that web images queried by action names serve as well-localized highlights for many actions, but are noisily labeled. To solve this problem, we propose a simple yet effective method that takes weak video labels and noisy image labels as input, and generates localized action frames as output. This is achieved by cross-domain transfer between video frames and web images, using pre-trained deep convolutional neural networks. We then use the localized action frames to train action recognition models with long short-term memory networks. We collect a fine-grained sports action data set FGA-240 of more than 130,000 YouTube videos. It has 240 fine-grained actions under 85 sports activities. Convincing results are shown on the FGA-240 data set, as well as the THUMOS 2014 localization data set with untrimmed training videos.
\end{abstract}

\section{Categories and Subject Descriptors}

I.2.10 [Vision and Scene Understanding]: Video analysis

\section{General Terms}

Algorithms, Experimentation, Measurement

\section{Keywords}

Fine-grained action localization, domain transfer, LSTM

\section{INTRODUCTION}

This paper addresses the problem of fine-grained action localization from unconstrained web videos. A fine-grained

Permission to make digital or hard copies of all or part of this work for personal or classroom use is granted without fee provided that copies are not made or distributed for profit or commercial advantage and that copies bear this notice and the full citation on the first page. Copyrights for components of this work owned by others than ACM must be honored. Abstracting with credit is permitted. To copy otherwise, or republish, to post on servers or to redistribute to lists, requires prior specific permission and/or a fee. Request permissions from Permissions@ acm.org. MM'15, October 26-30, 2015, Brisbane, Australia.

Copyright is held by the owner/author(s). Publication rights licensed to ACM. ACM 978-1-4503-3459-4/15/10 ...\$15.00.

DOI: http://dx.doi.org/10.1145/2733373.2806226

\section{YouTube}
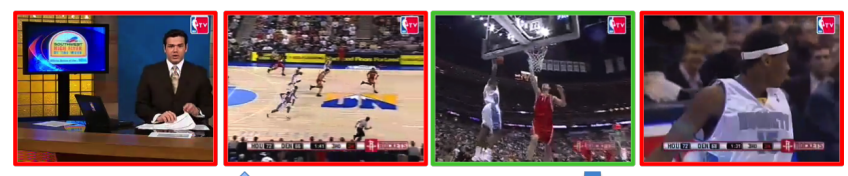

Cross-domain Transfer
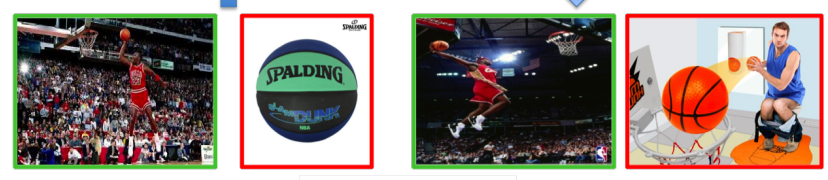

Google

Figure 1: Fine-grained actions are usually present as a tiny fraction within videos (top). Our framework uses cross-domain transfer from possibly noisy image search results (bottom) and identifies the action related images for both domains (marked in green).

action takes place in a higher-level activity or event (e.g., jump shot and slam dunk in basketball, blow candle in birthday party). Its instances are usually temporally localized within the videos, and share similar context with other finegrained actions belonging to the same activity or event.

Most existing work on action recognition focuses on action classification using pre-segmented short video clips 25, 14 23., which assumes implicitly that the actions of interest are temporally segmented during both training and testing. The TRECVID Multimedia Event Recounting evaluation [17] as well as THUMOS 14 Challenge 10 both address action localization in untrimmed video, but the typical approach involves training classifiers on temporally segmented action clips and testing using sliding window on untrimmed video. This setting does not scale to large action vocabularies, when data is collected from consumer video websites. Videos here are unconstrained in length, format (home videos vs. professional videos), and almost always only have video level annotations of actions.

We assume that only video-level annotations are available for the fine-grained action localization problem. The ability to localize fine grained actions in videos has important applications such as video highlighting, summarization, and automatic video transcription. It is also a challenging prob- 
lem for several reasons: first, fine-grained actions for any high-level activity or event are inherently similar since they take place in similar scene context; second, occurrences of the fine-grained actions are usually short (a few seconds) in training videos, making it difficult to associate the videolevel labels to the occurrences.

Our key observation is that one can exploit web images to help localize fine-grained actions in videos. As illustrated in Figure 1, by using action names (basketball slam dunk) as queries, many of the image search results offer well localized actions, though some of them are non-video like or irrelevant. Identifying action related frames from weakly supervised videos and filtering irrelevant image tags is hard in either modality by itself; however, it is easier to tackle these two problems together. This is due to our observation that although most of the video frames and web images which correspond to actions are visually similar, the distributions of non-action images from the video domain and the web image domain are usually very different. For example, in a video with a basketball slam dunk, non slam dunk frames in the video are mostly from a basketball game. The irrelevant results returned by image search are more likely to be product shots, or cartoons.

This motivates us to formulate a domain transfer problem between web images and videos. To allow domain transfer, we first treat the videos as a bag of frames, and use the feature activations from deep convolutional neural networks $(\mathrm{CNN})$ 13] as the common representation for images and frames. Suppose we have selected a set of video frames and a set of web images for every action, the domain transfer framework goes in two directions: video frames to web images, and vice versa. For both directions, we use the selected images from the source domain to train action classifiers by fine-tuning the top layers of the CNN; we then apply the trained classifiers to the target domain. Each image in the target domain is assigned a confidence score given by its associated action classifier from the source domain. By gradually filtering out the images with low scores, the bidirectional domain transfer can progress iteratively. In practice, we start from the video frames to web images direction, and randomly select the video frames for training. Since the non-action related frames are not likely to occur in web images, the tuned CNN can be used to filter out the non-video like and irrelevant web images. The final domain transfer from web images is used to localize action related frames in videos. We term these action-related frames as localized action frames (LAF).

Videos are more than an unordered collection of frames. We choose long short-term memory (LSTM) [8] networks as the temporal model. Compared with the traditional recurrent neural networks (RNN), LSTM has built-in input gates and forget gates to control its memory cells. These gates allow LSTM to either keep a long term memory or forget its history. The ability to learn from long sequences with unknown size of background is well-suited for fine-grained action localization from unconstrained web videos. We treat every sampled video frame as a time step in LSTM. When we train LSTM models, we label all video frames by their video-level annotation, but use the LAF scores generated by bidirectional domain transfer as weights on the loss for misclassification. By doing this, irrelevant frames are effectively down-weighted in the training stage. The framework can be naturally extended to use video shots as time steps, from which spatio-temporal features can be extracted to capture local motion information.

Fine-grained action localization from untrimmed web videos is a new task. The closest existing data set is THUMOS 2014 with 20 sports categories. It is designed for action localization using segmented videos as training, but has 1,010 untrimmed validation videos. To evaluate the framework in a large scale setting, we collected a new data set from YouTube. We chose 240 fine-grained actions belonging to 85 different sports activities, the total number of videos is over 130,000. Although the evaluated categories are sports actions, this method can be easily extended to other domains. For example, one can easily get cut cake, eat cake and blow candle images for a birthday party event with image search.

Our work makes three major contributions:

- We show that learning temporally localized actions from videos becomes much easier if we combine weakly labeled video frames and noisily tagged web images. This is achieved by a simple yet effective domain transfer algorithm.

- We propose a localization framework that uses LSTM network with the localized action frames to model the temporal evolution of actions.

- We introduce the problem of fine-grained action localization with untrimmed videos, and collect a large fine-grained sports action data set with over 130,000 videos in 240 categories. The data set is available online 1

\section{RELATED WORK}

Most existing work on activity recognition focuses on classifying pre-segmented clips. For example, UCF 101 data set 25] and HMDB 51 data set [14] provide 101 and 51 activity categories respectively. Activity types range from primitive human actions, sports to playing instruments; the typical length of each video clip is 5 to 10 seconds. More recently, Karpathy et al. 11 proposed the Sports-1M data set with more than 1 million untrimmed YouTube videos. Even though it offers 487 sports categories, most of them are high-level activities such as basketball and cricket. For finegrained action recognition, Rohrbach et al. 20] collected a cooking action data set with temporal annotation, the videos were shot in an indoor kitchen with static camera. To the best of our knowledge, there is no previous work on finegrained action localization with untrimmed training videos.

Action recognition typically involves two basic steps: feature extraction and classifier training. The standard approach is to extract hand-designed low-level features, and then aggregate the features into fixed-length feature vectors for classification. Oneata et al. 16 showed that a combination of visual features (SIFT 15]) and motion features (DT [31]) represented using Fisher Vectors [18] produced state-of-the-art activity and event classification performance.

Recent approaches, particularly those based on deep neural networks, jointly learn features and classifiers. Karpathy et al. 11 proposed several variations of convolutional neural net (CNN) architectures that extended Krizhevsky et al.'s

\footnotetext{
1 https://sites.google.com/site/finegrainedactions/
} 

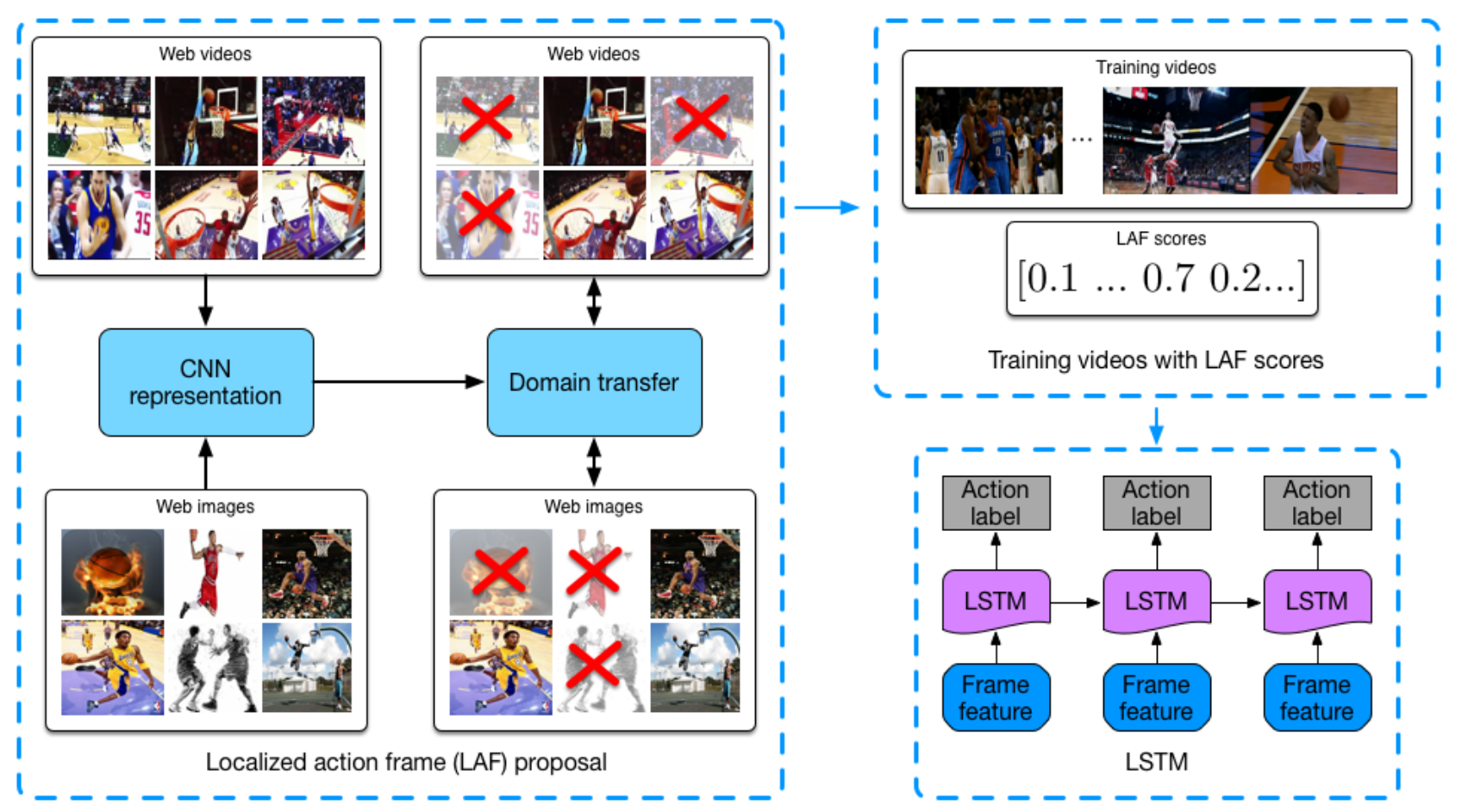

Figure 2: Illustration of the LAF proposal framework for basketball slam dunk videos. We use a cross-domain transfer algorithm to jointly filter out non-video like web images and non-action like video frames. We then learn a LAF proposal model using the filtered images, which assigns LAF scores to training video frames. Finally, we train LSTM based fine-grained action detectors, where the misclassification penalty of each time step is weighted by the LAF score.

image classification model 13 and attempted to learn motion patterns from spatio-temporal video patches. Simonyan and Zisserman 24] obtained good results on action recognition using a two-stream CNN that takes pre-computed optical flow as well as raw frame-level pixels as input.

There have been many attempts to address the action localization problem. Tian et al. 29 proposed a temporal extension of the deformable part model (DPM) for action detection, they used spatio-temporal bounding boxes for training. Wang et al. 33 used dynamic poselets which took motion and pose into account. Jain et al. 9] localized the actions with tubelets. All these approaches require manually annotated spatio-temporal bounding boxes for training. For temporal localization, THUMOS 2014 [10] data set provides trimmed video segments to train action classifiers, the classifiers can be used for localization with temporal sliding windows.

The idea of using images as auxiliary data has also recently been explored. The most common usage is to learn mid-level concept detectors for high-level event classification [1, 7, 27]. Yang et al. 36 propose a domain adaptation algorithm from images to videos, but assume perfect image annotation. Divvala et al. 3 learn mixtures of object sub-categories using web images, they achieving this goal by filtering sub-categories with low classification performance.

The long short-term memory network (LSTM) was proposed by Hochreiter and Schmidhuber 8] as an improvement over traditional recurrent neural networks (RNN) for classification and prediction of time series data. Specifically, an LSTM can remember and forget values from the past, unlike a regular RNN where error gradients decay exponentially quickly with the time lag between events. It has recently shown excellent performance in modeling sequential data such as speech recognition [22, 5, handwriting recognition [6] and machine translation 28]. More recently, LSTM has also been applied to video-level action classification 4 . 26, 38, and generate image descriptions 30, 12.

\section{OUR APPROACH}

Our proposed fine-grained action localization framework uses both weakly labeled videos and noisily tagged web images. It employs the same CNN based representation for web images and video frameworks, and uses a bidirectional domain transfer algorithm to filter out irrelevant images in both domains. A localized action frame (LAF) proposal model is trained from the remaining web images, and used to assign LAF scores to video frames. Finally, we use long short-term memory networks to train fine-grained action detectors, using the LAF scores as the weight of loss for misclassification. The pipeline is illustrated in Figure 2 .

\subsection{Shared CNN Representation}

A shared feature space is required for domain transfer between images and videos. Here we treat a video as a bag of frames, and extract activations from the intermediate layers of a convolutional neural network (CNN) as features for both web images and video frames. Although there is previous work on action recognition from still images using 


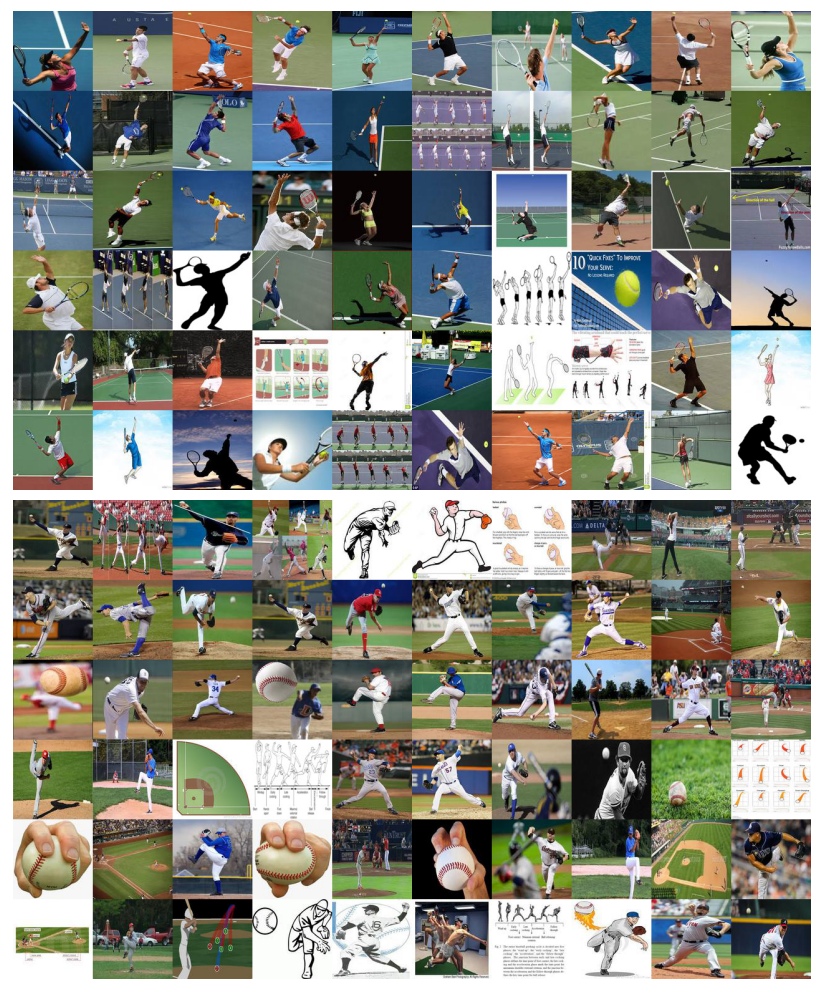

Figure 3: Top retrieved images from the Google image search engine, using keywords tennis serve and baseball pitch.

other representations 37], we choose CNN activations for its simplicity and state-of-the-art performance in several action recognition tasks 24,11 .

Training a CNN end-to-end from scratch is time consuming, and requires a large amount of annotated data. It has been shown that CNN weights trained from large image data sets like ImageNet 2] are generic, and can be applied to other image classification tasks by fine-tuning. It is also possible to disable the error back-propagation for the first several layers during fine-tuning. This is equivalent to training a shallower neural network using the intermediate CNN activations as features.

In this paper, we adopt the methodology of fine-tuning the top layers of CNN, and experiment with the AlexNet 13 CNN architecture. It contains five convolution layers and three fully connected layers. Each convolution layer is followed by a ReLU non-linearity layer and a maximum pooling layer. We pre-trained the network on ImageNet data set using the data partitions defined in 21]. We resized the images to 256 by 256 , and used the raw pixels as inputs. For the purpose of fine-tuning, we fixed the network weights before its first fully connected layer and only updated the parameters of the top three layers. Feature activations from fc6 serve as the shared representation for web images and video frames, and allow cross-domain transfer between the two.

\subsection{LAF Proposal with Web Images}

Fine-grained actions tend to be more localized in videos than high-level activities. For example, a basketball match video usually consists of jump shot, slam dunk, free throw etc., each of which may be as short as a few seconds. We address the problem of automatically identifying them from minutes-long videos.

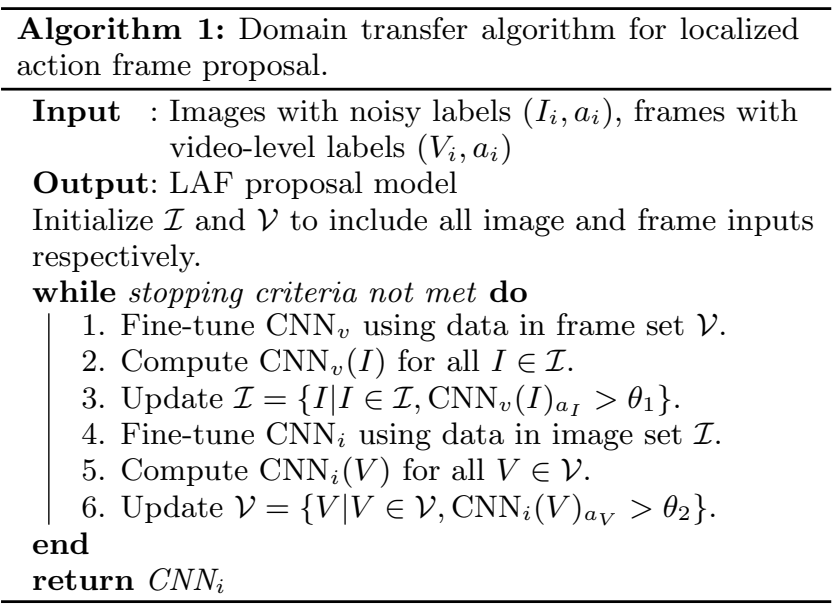

Fortunately, we observe that many of the fine-grained actions have image highlights on the Internet (Figure 3). They are easily obtained by querying image search engines with action names. However, these images are noisily labeled, and not useful for learning LAF proposal models directly, as they contain:

- Irrelevant images due to image crawling error, for example, a jogging image could be retrieved with the keyword soccer dribbling.

- Items related to the actions, such as objects and logos.

- Images with the same action but from a different domain, such as advertisement images with clear background, or cartoons.

Filtering the irrelevant web images is a challenging problem by itself. However, it can be turned into an easier problem by using weakly-supervised videos. We hypothesize that applying a classifier, learned on video frames, as a filter on the images removes many irrelevant images and preserves most video-like image highlights. More formally, assume we have video frames in $\mathcal{V}$ and web images in $\mathcal{I}$, and each of them is assigned a fine-grained action label $a=0,1, \ldots, N-1$. We first learn a multi-class classifier $\mathrm{CNN}_{v}(\cdot) \in \mathbb{R}^{N}$ by finetuning the top layers of $\mathrm{CNN}$ using video frames. $\mathrm{CNN}_{v}(\cdot)$ encodes action discriminative information from the videos' perspective; we apply it to all $I \in \mathcal{I}$, and update

$$
\mathcal{I}=\left\{I \mid I \in \mathcal{I}, \operatorname{CNN}_{v}(I)_{a_{I}}>\theta_{1}\right\}
$$

where $\theta_{1} \in[0,1]$ is the threshold for minimum softmax output, and $\mathrm{CNN}_{v}(I)_{a_{I}}$ corresponds to the $a_{I}$-th dimension of $\mathrm{CNN}_{v}(I)$.

We then use the filtered $\mathcal{I}$ to fine-tune $\operatorname{CNN}_{i}(\cdot) \in \mathbb{R}^{N}$, and update $\mathcal{V}$ in a similar manner:

$$
\mathcal{V}=\left\{V \mid V \in \mathcal{V}, \mathrm{CNN}_{i}(V)_{a_{V}}>\theta_{2}\right\}
$$

We iterate the process and update $\mathcal{V}$ and $\mathcal{I}$ until certain stopping criteria are met. The LAF proposal model $\mathrm{CNN}_{i}(\cdot)$ 
is learned using the final web image set $\mathcal{I}$, the LAF score for a video frame $V$ with action label $a$ is given by

$$
\operatorname{LAF}(V)=\mathrm{CNN}_{i}(V)_{a}
$$

The whole process is summarized in Algorithm 1

Discussion: We initialize the frame set $\mathcal{V}$ by random sampling. Even though many of the sampled frames do not correspond to the actions of interest, they can help filter out the non-video like web images such as cartoons, object photos and logos. In practice, the random sampling of video frames is adequate for this step since the mis-labeled frames rarely appear in the web image collection.

We set the stopping criteria to be: (1) video-level classification accuracy on a validation set starts to drop; or (2) a maximum number of iterations is reached. To be more efficient, we train one-vs-rest linear SVMs using frames in $\mathcal{V}$ after each iteration, and apply the classifiers to video frames in the validation set. We take the average of frame-level classifier responses to generate video-level responses, and use them to compute classification accuracy.

\subsection{Long Short-Term Memory Network}

Long Short-term Memory (LSTM) 8] is a type of recurrent neural network (RNN) that solves the vanishing and exploding gradients problem of previous RNN architectures when trained using back-propagation. Standard LSTM architecture includes an input layer, a recurrent LSTM layer and an output layer. The recurrent LSTM layer has a set of memory cells, which are used to store real-valued state information from previous observations. This recurrent information flow, from previous observations, is particularly useful for capturing temporal evolution in videos, which we hypothesize is useful in distinguishing between fine-grained sports activities. In addition, LSTM's memory cells are protected by input gates and forget gates, which allow it to maintain a long-term memory and reset its memory, respectively. We employ the modification to LSTMs proposed by Sak et al. 22 to add a projection layer after the LSTM layer. This reduces the dimension of stored states in memory cells, and helps to make the training process faster.

Let us denote the input sequence $\mathbf{X}$ as $\left\{x_{1}, x_{2}, \ldots, x_{T}\right\}$, where in our case each $x_{t}$ is a feature vector of a video frame with time stamp $t$. LSTM maps the input sequence into the output action responses $\mathbf{Y}=\left\{y_{1}, y_{2}, \ldots, y_{T}\right\}$ by:

$$
\begin{aligned}
i_{t} & =\sigma\left(W_{i x} x_{t}+W_{i r} r_{t-1}+W_{i c} c_{t-1}+b_{i}\right) \\
f_{t} & =\sigma\left(W_{f x} x_{t}+W_{r f} r_{t-1}+W_{c f} c_{t-1}+b_{f}\right) \\
c_{t} & =f_{t} \odot c_{t-1}+i_{t} \odot g\left(W_{c x} x_{t}+W_{c r} r_{t-1}+b_{c}\right) \\
o_{t} & =\sigma\left(W_{o x} x_{t}+W_{o r} r_{t-1}+W_{o c} c_{t}+b_{o}\right) \\
m_{t} & =o t \odot h\left(c_{t}\right) \\
r_{t} & =W_{r m} m_{t} \\
y_{t} & =W_{y r} r_{t}+b_{y}
\end{aligned}
$$

Here $W$ 's and $b$ 's are the weight matrices and biases, respectively, and $\odot$ denotes the element-wise multiplication operation. $c$ is the memory cell activation; $i, f, o$ are input gate, forget gate and output gate respectively. $m$ and $r$ are recurrent activation before and after projection. $\sigma$ is the sigmoid function, $g$ and $h$ are tanh. An illustration of the LSTM architecture with a single memory block is shown in Figure 4

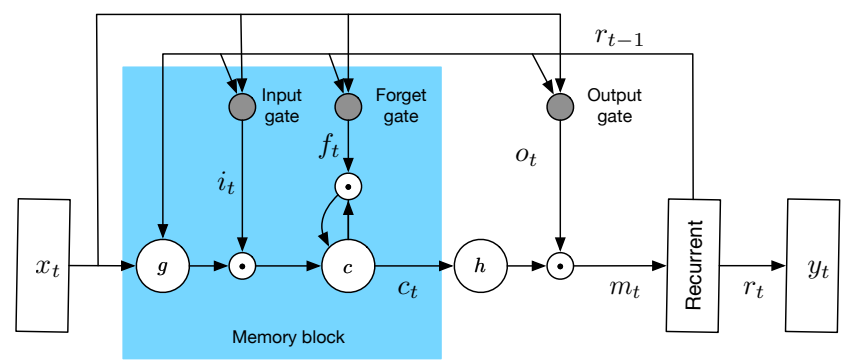

Figure 4: Illustration of LSTM architecture with a single memory block. A recurrent projection layer is added to reduce the number of parameters. Reproduced from [22] with the authors' permission.

Training LSTM with LAF scores. We sample video frames at 1 frame per second and treat each frame as a basic LSTM step. Similar to speech recognition tasks, each time step requires a label and a penalty weight for misclassification. The truncated backpropagation through time (BPTT) learning algorithm 34 is used for training. We limit the maximum unrolling time steps to $k$ and only back-propagate the error for $k$ time steps. Incorporating the LAF scores into the LSTM framework is simple: we first run the LAF proposal pipeline to score all sampled training video frames. Then we set the frame-level labels based on video-level annotation, but use the LAF scores as the penalty weights. Using this method, LSTM is forced to make the correct decision after watching a LAF returned by LAF proposal system, and it is not penalized as heavily when gathering context information from earlier frames or misclassifying an unrelated frame.

Computing LAF scores for video shots. For some data sets, it might be desirable to use video shots as the basic LSTM steps, as it allows the use of spatio-temporal motion features for representation. We extend the framelevel LAF scores to shot-level by taking the average of LAF scores from the sampled frames within a certain video shot.

\section{EXPERIMENTS}

This section first describes the data set we collected for evaluation, and then presents experimental results.

\subsection{Data Set}

There is no existing data set for fine-grained action localization using untrimmed web videos. To evaluate our proposed method's performance, we collected a Fine Grained Actions 240 (FGA-240) data set focusing on sports videos. It consists of over 130,000 YouTube videos in 240 categories. A subset of the categories is shown in Figure 5 We selected 85 high-level sports activities from the Sports-1M data set 11, and manually chose the fine-grained actions take place in these activities. The action categories cover aquatic sports, team sports, sports with animals and others.

We decided the fine-grained categories for each high-level sports activity using the following method: given YouTube videos and their associated text data such as titles and descriptions, we ran an automatic text parser to recognize sports related entities. The recognized entities which correlate with the high-level sports activities were stored in the 


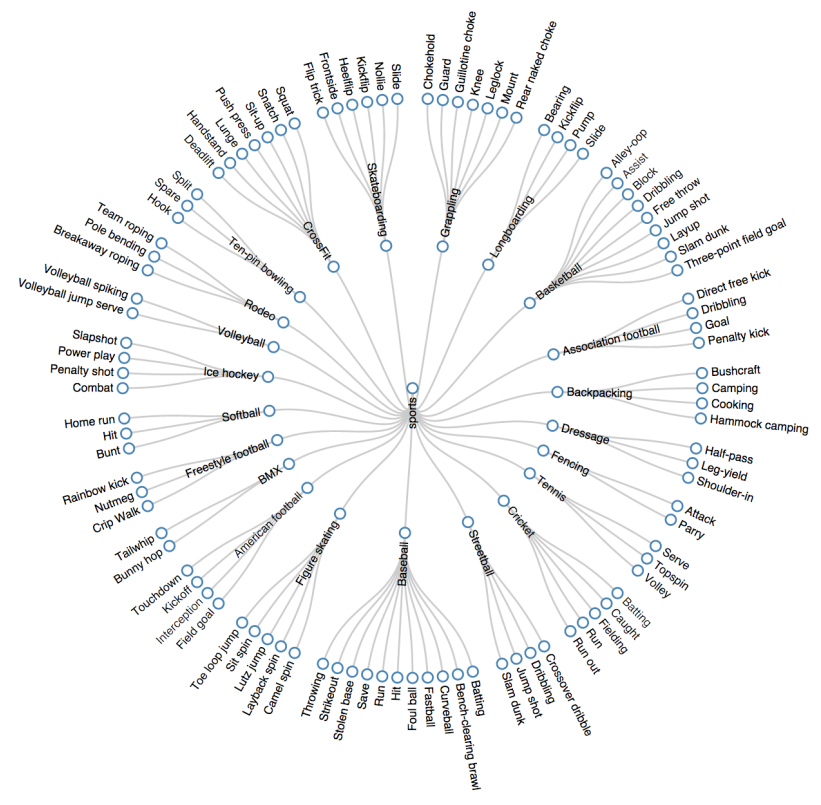

Figure 5: Some of the high-level sports activities and their corresponding fine-grained sports actions in Fine Grained Actions 240 data set. Best viewed under magnification.

pool and then manually filtered to keep only fine-grained sports actions. As an example, for basketball the initial entity pool contains not only fine-grained sports actions (e.g., slam dunk, block), but also game events (e.g., NBA) and celebrities (e.g., Kobe Bryant). Once the fine-grained categories are fixed, we applied the same text analyzer to automatically assign video-level annotations, and only kept the videos with high annotation confidence. We finally visualized the data set to filter out false annotations and removed the fine-grained sports action categories with too few samples.

Our final data set contains 48,381 training videos and 87,454 evaluation videos. The median number of training videos per category is 133 . We used $20 \%$ of the evaluation videos for validation and $80 \%$ for testing.

For temporal localization evaluation, we manually annotated 400 videos from 45 fine-grained actions. The average length of the videos is 79 seconds.

\subsection{Experiment Setup}

LSTM implementation. We used the feature activations from pre-trained AlexNet (first fully-connected layer with 4,096 dimensions) as the input features for each time step. We followed the LSTM implementation by Sak et al. 22 which utilizes a multi-core CPU on a single machine. For training, we used asynchronous stochastic gradient descent and set batch size to 12 . We tuned the training parameters on the validation videos and set the number of LSTM cells to 1024 , learning rate to 0.0024 and learning rate decay with a factor of 0.1 . We fixed the maximum unroll time step $k$ to 20 to forward-propagate the activations and backwardpropagate the errors.
Video level classification. We evaluated fine-grained action classification results on video level. We sampled test video frames at 1 frame per second. Given $T$ sampled frames from a video, these frames are forward-propagated through time, and produce $T$ softmax activations. We used average fusion to aggregate the frame-level activations over whole videos.

Temporal localization. We generated the frame-level softmax activations using the same approach as video level classification. We used a temporal sliding window of 10 time steps, the score of each sliding window was decided by taking the average of softmax activations. We then applied non-maximum suppression to remove the localized windows which overlap with each other.

Evaluation metric. For classification, we report Hit @ $k$, which is the percentage of testing videos whose labels can be found in the top $k$ results. For localization, we follow the same evaluation protocol as THUMOS 2014 [10] and evaluate mean average precision. A detection is considered to be a correct one if its overlap with groundtruth is over some ratio $r$.

CNN baseline. We deployed the single-frame architecture used by Karpathy et al. 11] as the CNN baseline. It was shown to have comparable performance with multiple variations of CNNs while being simpler. We sampled the video frames at 1 frame per second, and used average fusion to aggregate softmax scores for classification and localization tasks. Instead of training a CNN from scratch, we used network parameters from the pre-trained AlexNet, and finetuned the top two fully-connected layers and a softmax layer. Training parameters were decided using the validation set.

Low-level feature baseline. We extracted low-level features used by 11, 35. over whole videos for classification task, the feature set includes low-level visual and motion features aggregated using bag-of-words. We used the same neural network architecture as 11 with multiple Rectified Linear Units to build classifiers based on the low-level features. Its structure (e.g., number of layers, number of cells per layer) as well as training parameters were decided with validation set.

\subsection{Video-level Classification Results}

We first report the fine-grained action classification performance on video level.

Comparison with baselines. We compared several baseline systems' performance against our proposed method on FGA-240 data set, the results are shown in Table 1. From the table we can see that systems based on CNN activations outperformed low-level features by a large margin. There are two possible reasons for this: first, CNN learned activations are more discriminative in classifying fine-grained sports actions, even without capturing local motion patterns explicitly; second, low-level features were aggregated on video-level. These video-level features are more sensitive to background and irrelevant video segments, which happens a lot in fine-grained sports action videos.

Among the systems relying on CNN activations, applying LSTM gave better performance than fine-tuning the top layers of CNN. While both LSTM and CNN used the late fusion of frame-level softmax activations to generate videolevel classification results, LSTM took previous observations into consideration with the help of memory cells. This shows 


\begin{tabular}{c|c|c}
\hline Method & Video Hit @1 & Video Hit @5 \\
\hline Random & 0.4 & 2.1 \\
\hline Low-level features 35 & 30.8 & - \\
\hline CNN 11. & 37.3 & 68.5 \\
\hline LSTM w/o LAF & 41.1 & 70.2 \\
LSTM w/ LAF & $\mathbf{4 3 . 4}$ & $\mathbf{7 4 . 9}$ \\
\hline
\end{tabular}

Table 1: Video-level classification performance of several different systems on fine-grained actions.
Videos
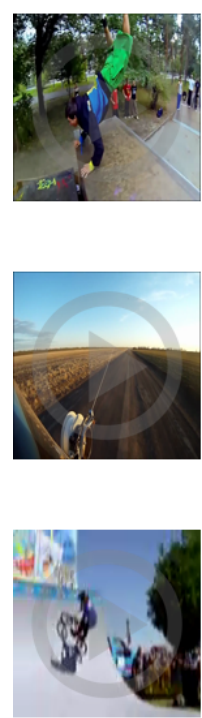

Web images
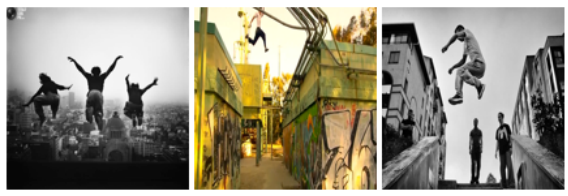

Parkour:Free Running

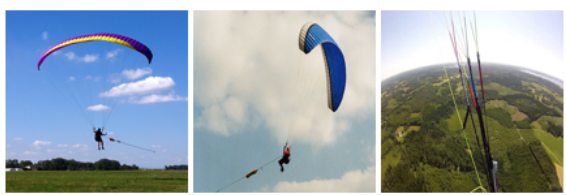

Paragliding: Towing

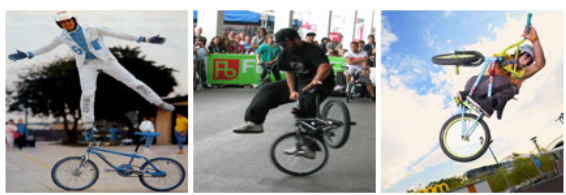

Freestyle BMX: Stunt
Figure 6: Example actions when LAF is not helpful. The web images retrieved to generate $\mathrm{LAF}$ proposals might be beautified (top) or taken from different viewpoint (middle). Sometimes there is a mix of the two issues (bottom).

that temporal information helps classify fine-grained sports actions, and it was captured by LSTM network.

Finally, using LAF proposals helped further improve the video hit @1 by $2.3 \%$ and video hit @ 5 by $4.7 \%$. In Table 2 we show the relative difference in average precision for LSTM with and without LAF proposal. We observe that LAF proposal helps the most when the fine-grained sports actions are likely to be identified based on single frames, and the image highlights on the Internet are visually very similar to the videos. Note that there are still non-video-like and irrelevant images retrieved from the Internet for these categories, but the LAF proposal system is an effective filter. Figure 8 gives the three systems' output on a few example videos.

We also identify several cases when LAF proposals failed to work. The most common case is when most of the retrieved images are non-video like but not filtered out. They could be posed images or beautified images with logos, such as images retrieved for Parkour:Free running, or have different viewpoints than videos, such as Paragliding:Towing.

\begin{tabular}{rr|cl}
\hline Fine-grained sports & $\Delta$ AP & $\Delta$ AP & Fine-grained sports \\
\hline Fencing:Parry & 0.17 & -0.09 & Parkour:Free Running \\
Cricket:Run out & 0.15 & -0.08 & Freestyle soccer:Crip Walk \\
CrossFit:Deadlift & 0.10 & -0.08 & Paragliding:Towing \\
CrossFit:Handstand & 0.09 & -0.07 & Freestyle BMX:Stunt \\
Calisthenics:Push-up & 0.09 & -0.07 & Judo:Sweep \\
Rings:Pull-up & 0.08 & -0.06 & Basketball:Point \\
\hline
\end{tabular}

Table 2: Difference in average precision between LSTM with and without LAF proposal. Sorted by top wins (left) and top losses (right).

\begin{tabular}{|c|c|c|}
\hline Method & Video Hit @1 & Video Hit @5 \\
\hline Random & 1.2 & 5.9 \\
\hline CNN 11 & 69.2 & 75.9 \\
\hline LSTM w o LAF & 71.7 & 77.3 \\
\hline LSTM w/ LAF & 73.6 & 79.5 \\
\hline
\end{tabular}

Table 3: Classification performance when measured on high-level sports activities (e.g., basketball, soccer).

Sample video snapshots and web images are shown in Figure 6.

Impact of action hierarchy. A fine-grained sports action could be misclassified to either its sibling or non-sibling leaf nodes in the sports hierarchy. For example, a basketball slam dunk can be confused with basketball alley-oop as well as street ball slam dunk. To study the source of confusion, we decided to measure classification accuracy of high-level sports activities, and check how the numbers compared with fine-grained sports actions.

We obtain the confidence values for high-level sports activities by taking the average of their child nodes' confidence scores. Table 3 shows the classification accuracy with different methods. We can see that the overall trend is the same as fine-grained sports actions: LSTM with LAF proposal is still the best. However, the numbers are much higher than when measured on fine-grained level, which indicates that the major source of confusion still comes from the finegrained level. In Figure 7] we provide the zoom-in confusion matrices for ice hockey, crossfit and basketball.

\subsection{Localization Results}

Comparison with baselines. We applied the frameworks to localize fine-grained actions, and varied the overlap ratio $r$ from 0.1 to 0.5 for evaluation. Figure 9 shows the mean average precision over all 45 categories of different systems. We did not include the baseline using low-level features for evaluation as they were computed over whole videos. From the figure we can see that LSTM with LAF proposal outperformed both CNN and LSTM without LAF proposal significantly, the gap grows wider as we increase the overlap ratio. This confirms that temporal information and LAF proposal are helpful for the temporal localization task.

In Table 4, we show the most different average precisions on action level. Some actions have clearly benefited from the introduction of LSTM as well as LAF proposal. We also observed that some actions were completely missed by all three systems, such as Baseball:Hit, Basketball:Three-point field goal and Basketball:Block, possibly due to the video 

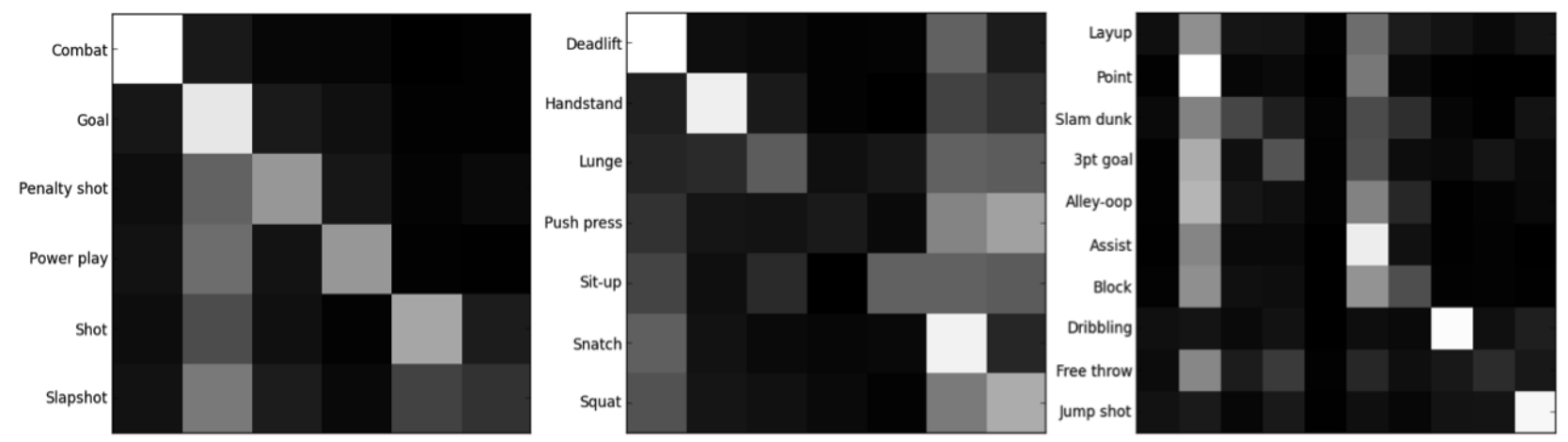

Figure 7: Magnified view of confusion matrices for ice hockey, crossfit and basketball
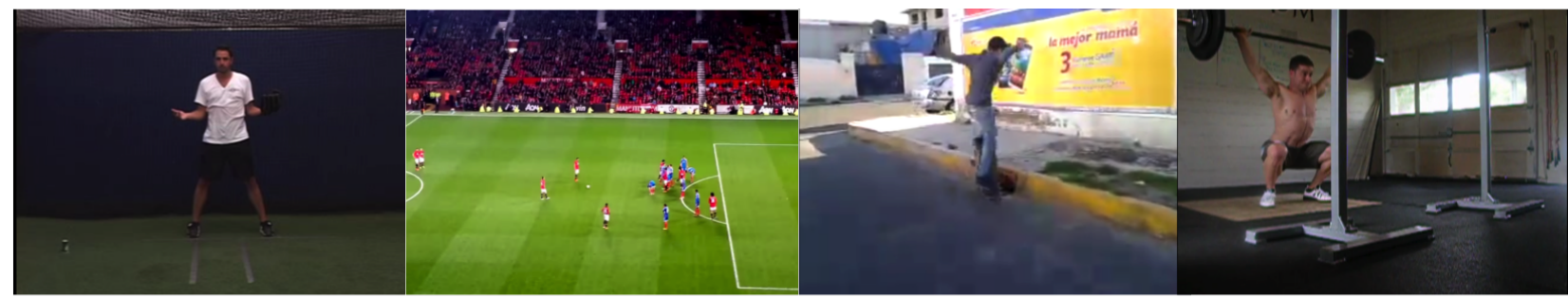

$\begin{array}{cccc}\text { Baseball:Throwing } & \text { Soccer:Direct freekick } & \text { Skateboarding:Nollie } & \text { CrossFit:Squat } \\ \text { Tennis:Forehand } & \text { American football:Kickoff } & \text { Longboarding:Slide } & \text { CrossFit:Sit-up } \\ \text { Tennis:Serve } & \text { Rugby union:Try } & \text { BMX:Flatland BMX } & \text { CrossFit:Snatch }\end{array}$
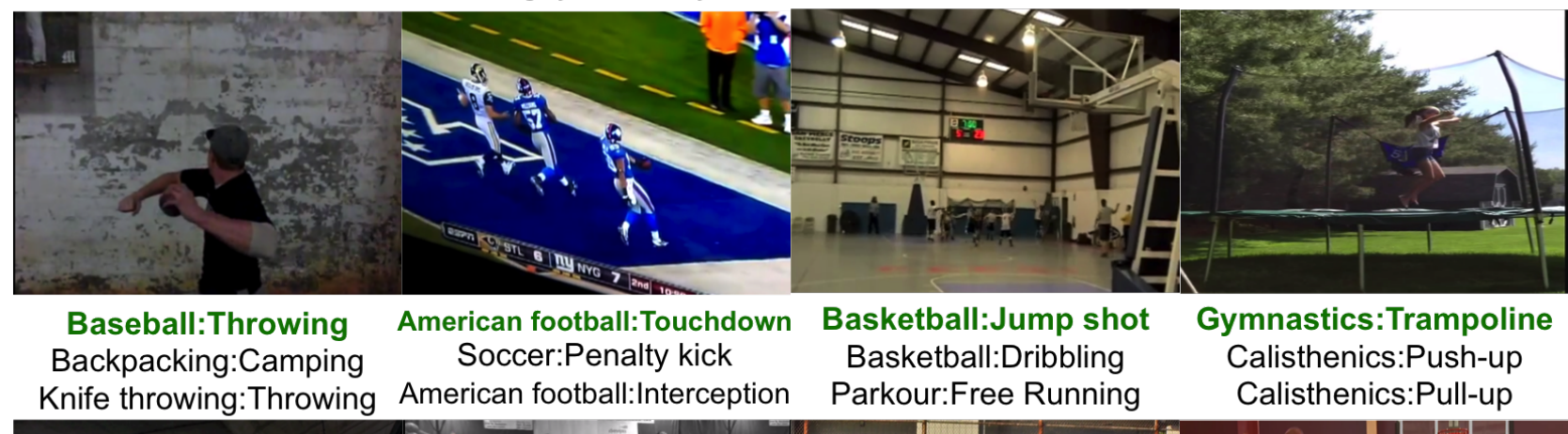

Gymnastics:Trampoline Calisthenics:Push-up

Calisthenics:Pull-up

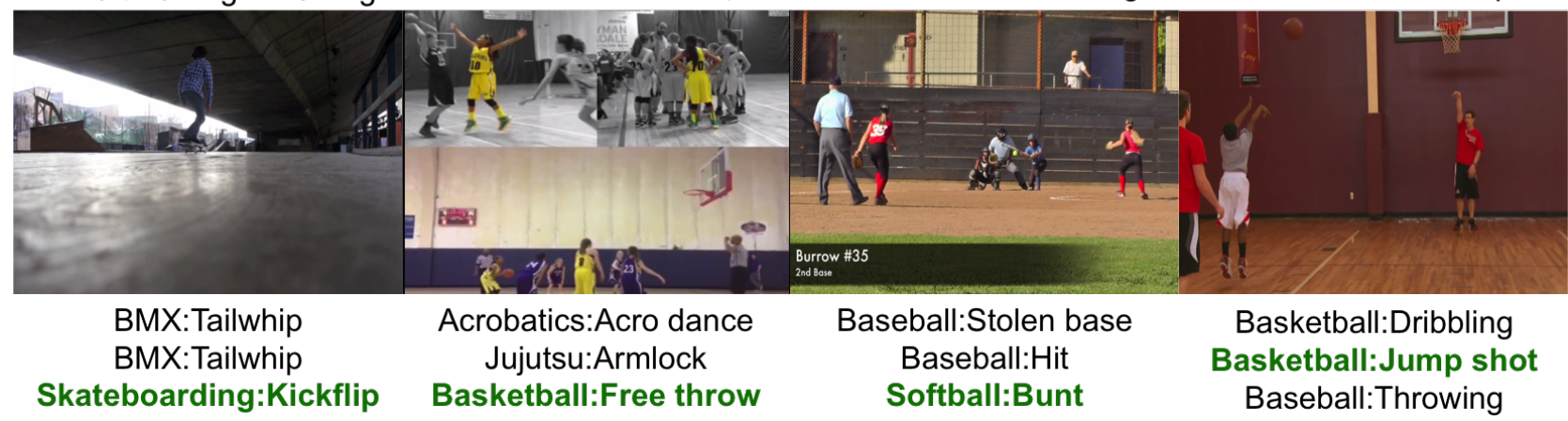

Figure 8: Classification output for a few videos. The labels under each video were generated by LSTM with LAF proposal, LSTM without LAF proposal and CNN from top to bottom. Correct answers are marked in bold. 


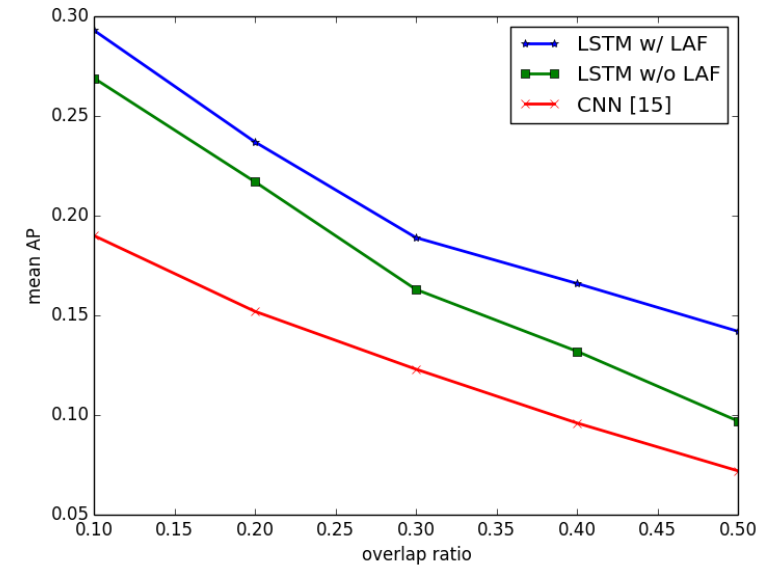

Figure 9: Temporal localization performance on FGA-240 data set.

\begin{tabular}{rr|ll}
\hline Fine-grained sports & $\Delta$ AP & $\Delta$ AP & Fine-grained sports \\
\hline Soccer:Penalty kick & 0.32 & -0.06 & Baseball:Run \\
Tennis:Serve & 0.25 & -0.01 & Skateboarding:Kickflip \\
Basketball:Dribbling & 0.21 & -0.01 & Volleyball:Spiking \\
\hline \multicolumn{4}{l}{} \\
\hline Fine-grained sports & $\Delta$ AP & $\Delta$ AP & Fine-grained sports \\
\hline Baseball:Brawl & 0.52 & -0.11 & Streetball:Crossovers \\
Ice hockey:Combat & 0.48 & -0.05 & Ice hockey:Penalty shot \\
Soccer:Penalty kick & 0.33 & -0.04 & Fencing:Parry \\
\hline
\end{tabular}

Table 4: Difference in average precision, compared between LSTM with and without LAF proposal (top), LSTM with LAF proposal and CNN (bottom), overlap ratio is fixed to 0.5. A positive number means LSTM with LAF proposal is better.

frames corresponding to these actions not being well localized during training.

\subsection{Localization Results on THUMOS 2014}

To verify the effectiveness of domain transfer from web images, we also conducted a localization experiment on the THUMOS 2014 data set 10 . This data set consists of over 13,000 temporally trimmed videos from 101 actions, 1,010 temporally untrimmed videos for validation and 2,574 temporally untrimmed videos for testing. The localization annotations cover 20 out of the 101 actions in the validation and test sets. All 20 actions are sports related.

Experiment setup: As this paper focuses on temporal localization of untrimmed videos, we dropped the 13,000 trimmed videos, and used the untrimmed validation videos as the only positive samples for training. We also used 2,500 background videos as the shared negative training data.

To generate LAF scores, we downloaded web images from Flickr and Google using the action names as queries. We also sampled training video frames at 1 frame per second. We used the AlexNet features for domain transfer experiment.

Recently, it has been shown that a combination of improved dense trajectory features 32 and Fisher vector encoding 18] (iDT + FV) offers the state-of-the-art performance

\begin{tabular}{c|ccccc}
\hline & \multicolumn{5}{|c}{ Overlap ratio } \\
\hline Method & 0.1 & 0.2 & 0.3 & 0.4 & 0.5 \\
\hline Ground truth & 0.161 & 0.152 & 0.112 & 0.071 & 0.044 \\
\hline Video 27 19 & 0.098 & 0.089 & 0.071 & 0.041 & 0.024 \\
LSTM w/o LAF & 0.076 & 0.071 & 0.057 & 0.038 & 0.024 \\
LSTM w/ LAF & $\mathbf{0 . 1 2 4}$ & $\mathbf{0 . 1 1 0}$ & $\mathbf{0 . 0 8 5}$ & $\mathbf{0 . 0 5 2}$ & $\mathbf{0 . 0 4 4}$ \\
\hline
\end{tabular}

Table 5: Temporal localization on the test partition of THUMOS 2014 dataset. Ground truth uses temporal annotation of the training videos.

on this data set. This motivated us to switch LSTM time steps from frames to video segments, and represent segments with iDT $+\mathrm{FV}$ features for the final detector training. We segmented all videos uniformly with a window width of 100 frames and step size of 50 frames. For iDT $+\mathrm{FV}$ feature extraction, we took only the MBH modality with 192 dimensions and reduced the dimensions to 96 with PCA. We used the full Fisher vector formulation with the number of GMM cluster centers set to 128 . The final video segment representation has 24,576 dimensions.

Results: We compared the performance of LSTM weighted by LAF scores against several baselines. LSTM w/o LAF randomly assigned misclassification penalty for each step of LSTM, where $30 \%$ of the steps were set to 1 , and others 0 . The Video baseline used iDT + FV features aggregated over whole videos to train linear SVM classifiers, and applied the classifiers to the testing video shots. It was used by 27 . 19] and achieved state-of-the-art performance in event recounting and video summarization tasks. None of these systems require temporal annotations. Finally, Ground truth employed manually annotated temporal localizations to set LSTM penalty weights. It is used to study the performance difference between LAF and an oracle with perfect localized actions.

Table 5 shows the mean average precision for the four approaches. As expected, using manually annotated ground truth for training provides the best localization performance. Although LSTM with LAF scores has worse performance than using ground truth, it outperforms LSTM without LAF scores, and the video-level baseline by large margins. This further confirms that LAF proposal by domain transfer from web images is effective in action localization tasks.

\section{CONCLUSION}

We studied the problem of fine-grained action localization for temporally untrimmed web videos. We proposed to use noisily tagged web images to discover localized action frames (LAF) from videos, and model temporal information with LSTM networks. We conducted thorough evaluations on our collected FGA-240 data set and the public THUMOS 2014 data set, and showed the effectiveness of LAF proposal by domain transfer from web images.

\section{ACKNOWLEDGMENTS}

We thank George Toderici, Matthew Hausknecht, Jia Deng, Weilong Yang, Susanna Ricco, Tomas Izo, Thomas Leung, Congcong $\mathrm{Li}$ and Howard Zhou for helpful comments and discussions. We also thank Bernard Ghanem, Fabian Caba Heilbron and Juan Carlos Niebles for kindly providing us video annotation tools. 


\section{REFERENCES}

[1] J. Chen, Y. Cui, G. Ye, D. Liu, and S. Chang. Event-driven semantic concept discovery by exploiting weakly tagged internet images. In ICMR, 2014.

[2] J. Deng, W. Dong, R. Socher, L.-J. Li, K. Li, and L. Fei-Fei. ImageNet: A Large-Scale Hierarchical Image Database. In CVPR, 2009.

[3] S. K. Divvala, A. Farhadi, and C. Guestrin. Learning everything about anything: Webly-supervised visual concept learning. In CVPR, 2014.

[4] J. Donahue, L. A. Hendricks, S. Guadarrama, M. Rohrbach, S. Venugopalan, K. Saenko, and T. Darrell. Long-term recurrent convolutional networks for visual recognition and description. In CVPR, 2015.

[5] A. Graves, A. Mohamed, and G. E. Hinton. Speech recognition with deep recurrent neural networks. In ICASSP, 2013.

[6] A. Graves and J. Schmidhuber. Offline handwriting recognition with multidimensional recurrent neural networks. In NIPS, 2008.

[7] A. Habibian, K. E. A. van de Sande, and C. G. M. Snoek. Recommendations for video event recognition using concept vocabularies. In ICMR, 2013.

[8] S. Hochreiter and J. Schmidhuber. Long short-term memory. Neural Computation, 1997.

[9] M. Jain, J. van Gemert, H. Jégou, P. Bouthemy, and C. G. M. Snoek. Action localization with tubelets from motion. In CVPR, 2014.

[10] Y.-G. Jiang, J. Liu, A. Roshan Zamir, G. Toderici, I. Laptev, M. Shah, and R. Sukthankar. THUMOS challenge: Action recognition with a large number of classes. http://crcv.ucf.edu/THUMOS14/, 2014.

[11] A. Karpathy, G. Toderici, S. Shetty, T. Leung, R. Sukthankar, and L. Fei-Fei. Large-scale video classification with convolutional neural networks. In CVPR, 2014.

[12] R. Kiros, R. Salakhutdinov, and R. S. Zemel. Unifying visual-semantic embeddings with multimodal neural language models. TACL, 2015.

[13] A. Krizhevsky, I. Sutskever, and G. E. Hinton. Imagenet classification with deep convolutional neural networks. In NIPS, 2012.

[14] H. Kuehne, H. Jhuang, E. Garrote, T. Poggio, and T. Serre. HMDB: a large video database for human motion recognition. In $I C C V, 2011$.

[15] D. G. Lowe. Distinctive image features from scale-invariant keypoints. IJCV, 2004.

[16] D. Oneata, J. Verbeek, and C. Schmid. Action and Event Recognition with Fisher Vectors on a Compact Feature Set. In ICCV, 2013.

[17] P. Over, G. Awad, M. Michel, J. Fiscus, G. Sanders, W. Kraaij, A. F. Smeaton, and G. Queenot. TRECVID 2013 - an overview of the goals, tasks, data, evaluation mechanisms and metrics. In TRECVID, 2013.

[18] F. Perronnin and C. Dance. Fisher kernels on visual vocabularies for image categorization. In CVPR, 2007.

[19] D. Potapov, M. Douze, Z. Harchaoui, and C. Schmid. Category-specific video summarization. In $E C C V$, 2014.
[20] M. Rohrbach, S. Amin, M. Andriluka, and B. Schiele. A database for fine grained activity detection of cooking activities. In CVPR, 2012.

[21] O. Russakovsky, J. Deng, H. Su, J. Krause, S. Satheesh, S. Ma, Z. Huang, A. Karpathy, A. Khosla, M. Bernstein, A. C. Berg, and L. Fei-Fei. ImageNet Large Scale Visual Recognition Challenge, 2014.

[22] H. Sak, A. Senior, and F. Beaufays. Long short-term memory based recurrent neural network architectures for large vocabulary speech recognition. CoRR, abs/1402.1128, 2014.

[23] C. Schuldt, I. Laptev, and B. Caputo. Recognizing human actions: A local SVM approach. In ICPR, 2004.

[24] K. Simonyan and A. Zisserman. Two-stream convolutional networks for action recognition in videos. In NIPS, 2014.

[25] K. Soomro, A. R. Zamir, and M. Shah. UCF101: A dataset of 101 human actions classes from videos in the wild. $C R C V-T R-12-01$.

[26] N. Srivastava, E. Mansimov, and R. Salakhutdinov. Unsupervised learning of video representations using LSTMs. In ICML, 2015.

[27] C. Sun, B. Burns, R. Nevatia, C. Snoek, B. Bolles, G. Myers, W. Wang, and E. Yeh. ISOMER: Informative segment observations for multimedia event recounting. In $I C M R, 2014$.

[28] I. Sutskever, O. Vinyals, and Q. V. Le. Sequence to sequence learning with neural networks. In NIPS, 2014.

[29] Y. Tian, R. Sukthankar, and M. Shah. Spatiotemporal deformable part models for action detection. In CVPR, 2013.

[30] O. Vinyals, A. Toshev, S. Bengio, and D. Erhan. Show and tell: A neural image caption generator. In $C V P R$, 2015.

[31] H. Wang, A. Kläser, C. Schmid, and C.-L. Liu. Dense trajectories and motion boundary descriptors for action recognition. IJCV, 2013.

[32] H. Wang and C. Schmid. Action Recognition with Improved Trajectories. In ICCV, 2013.

[33] L. Wang, Y. Qiao, and X. Tang. Video action detection with relational dynamic-poselets. In $E C C V$, 2014.

[34] R. J. Williams and J. Peng. An efficient gradient-based algorithm for on-line training of recurrent network trajectories. Neural Computation, 1990.

[35] W. Yang and G. Toderici. Discriminative tag learning on youtube videos with latent sub-tags. In $C V P R$, 2011.

[36] Y. Yang, Y. Yang, and H. T. Shen. Effective transfer tagging from image to video. TOMM, 2013.

[37] B. Yao and F. Li. Recognizing human-object interactions in still images by modeling the mutual context of objects and human poses. PAMI, 2012.

[38] J. Yue-Hei Ng, M. Hausknecht, S. Vijayanarasimhan, O. Vinyals, R. Monga, and G. Toderici. Beyond short snippets: Deep networks for video classification. In CVPR, 2015. 